\title{
Alzheimer's Disease and Memory Strength: Gradual Decline of Memory Traces as a Function of
}

\section{their Strength}

\author{
Guillaume T. Vallet ${ }^{1,2}$ \\ gtvallet@gmail.com \\ +1 (514) 340-3540 \#4140
}

CRIUGM, 4545 Chemin Queen-Mary, Montreal (Qc) H3W1W4 (Canada)

$$
\begin{gathered}
\text { Isabelle Rouleau } \\
\text { rouleau.isabelle@uqam.ca } \\
\text { +1 (514) } 9873000 \text { \#8915 }
\end{gathered}
$$

UQAM, SU1365 au 200 rue Sherbrooke ouest, Montreal (Qc) H3C 3P8 (Canada)

Sophie Benoit ${ }^{3} \&$ Roxane Langlois ${ }^{3}$

so.benoit@hotmail.com \& roxane.langlois@umontreal.ca

+1 (514) $9873000 \# 8915$

QAM, SU1365 au 200 rue Sherbrooke ouest, Montreal (Qc) H3C 3P8 (Canada)

\section{Emmanuel J. Barbeau ${ }^{5}$}

emmanuel.barbeau@cerco.ups-tlse.fr

$$
\text { +33(0)562746155 }
$$

CERCO, Pavillon Baudot, CHU Purpan, BP 25202, 31052 Toulouse Cedex (France)

$$
\begin{gathered}
\text { Sven Joubert }{ }^{1,2} \\
\text { sven.joubert @ umontreal.ca } \\
+1 \text { (514) 340-3540 \#4140 }
\end{gathered}
$$

CRIUGM, 4545 Chemin Queen-Mary, Montreal (Qc) H3W1W4 (Canada)

${ }^{1}$ Centre de recherche de l'IUGM, Université de Montréal, Montréal (Qc, Canada)

${ }^{2}$ Département de psychologie, Université de Montréal (Qc, Canada)

${ }^{3}$ Département de psychologie, Université du Québec à Montréal, Montréal (Qc, Canada)

${ }^{4}$ Centre de recherche, Centre hospitalier de l'Université de Montréal, Montréal (Qc, Canada)

${ }^{5}$ Université de Toulouse, UPS, Centre de Recherche Cerveau et Cognition, CNRS, CerCo, Toulouse (France) 


\begin{abstract}
Objective: Episodic memory impairment is at the core of amnestic Mild cognitive impairment (aMCI) and Alzheimer's disease (AD). The origin of memory deficits may result either from an encoding deficit or from an accelerated decline of the memory trace. The present study explores these two hypotheses.
\end{abstract}

Method: We used the DMS-48 (i.e., Delayed-Matching-to Sample 48 items) memory test in a group of controls, aMCI patients and AD patients ( $\mathrm{n}=16$ in each group). The DMS-48 offers an incidental learning phase followed by three forced-choice recognition tests at three-minute, one-hour and oneweek delays. Moreover, the forced-choice test distinguishes three kinds of couple items: abstract (meaningless), paired (two similar exemplars), and unique (two different objects) items.

Results: As predicted by the accelerated forgetting hypothesis, patients showed a decrease in recognition performance over time. Controls also exhibited a similar decline in performance. As predicted by the encoding deficit hypothesis, abstract items were the most poorly recognized in AD, at both the three minute and one-week delays. In $\mathrm{AD}$, recognition of the paired items also dropped after the one-hour delay, followed by unique items after a one-week delay. Patients with aMCI exhibited a performance that was similar to controls, except for abstract items, which dropped at the one-week delay.

Conclusions: These results are discussed in light of a third hypothesis, the memory strength hypothesis, in order to better account for the progressive decline in memory performance as a function of the item type in AD.

Keywords: episodic memory; Alzheimer's disease; amnestic mild cognitive impairment; encoding deficit; rapid forgetting; memory strength 


\section{Alzheimer's Disease and Memory Strength: Gradual Decline of Memory Traces as a Function of their Strength}

Episodic memory designates the personal memories of an individual grounded in a specific temporal and spatial context (Tulving, 1983, 2002). Memory impairment is at the core of Alzheimer's disease (AD), and of amnestic Mild cognitive impairment (aMCI), considered by many authors to be a transitional stage between normal aging and AD (Bäckman, Jones, Berger, Laukka, \& Small, 2005; Stopford, Snowden, Thompson, \& Neary, 2007). In aMCI and AD, encoding, storing and retrieval processing are typically impaired, although the degree of impairment is less pronounced in aMCI (Greenaway et al., 2006; see also Bennett, Golob, Parker, \& Starr, 2006) than in AD (Fleischman \& Gabrieli, 1999).

The source of the memory deficits remains nonetheless openly debated (e.g., Ally, Hussey, Ko, \& Molitor, 2013). Among the different hypotheses proposed (see Carlesimo \& Oscar-Berman, 1992), the accelerated rate of forgetting hypothesis and the encoding deficit hypothesis appear to be the most consensual. The accelerated rate of forgetting hypothesis implies that memory impairment in AD finds its origin in the hastened decline of the memory trace. For its part, the encoding deficit hypothesis states that $\mathrm{AD}$ patients are unable to effectively encode new memories and hence to retrieve them later (Larrabee, Youngjohn, Sudilovsky, \& Crook, 1993). The accelerated forgetting hypothesis also finds evidence in aMCI (Crowell, Luis, Vanderploeg, Schinka, \& Mullan, 2002). In one study, aMCI patients showed a memory decline as soon as 30 min after the immediate recall tests, but they exhibited a more pronounced decline in memory performance than controls at a 6- week than at a 30-min delay (Manes, Serrano, Calcagno, Cardozo, \& Hodges, 2008). This decline was observed for both verbal and visual material in free recall tasks. As in AD, a consolidation impairment was found for aMCI even after controlling for encoding (threshold of 90\% correct recognition, Walsh et al., 2014). 
The question of the delay at which memory decline accelerates remains to be determined. Some studies reported rapid forgetting after short delays in AD (10-min delay; Hart, Kwentus, Harkins, \& Taylor, 1988), followed by normalization (comparable slopes between patients and controls) or stabilization of the memory performance (Carlesimo, Fadda, Bonci, \& Caltagirone, 1993). In contrast, other studies reported accelerated forgetting even three months after initial learning (Budson et al., 2004). In this study, memory performance also appeared to stabilize over time since the rate of decline became similar across groups between the three-month and one-year delays (Budson et al., 2007).

Finally, in support of the rapid forgetting hypothesis, it has been consistently found that delayed memory is the most sensitive and specific index (receiver-operating characteristic, ROC analyses) to discriminate between AD, aMCI, and healthy aging after a 10-min (e.g., Welsh, Butters, Hughes, Mohs, \& Heyman, 1992) or 30-min delay (e.g., Estévez-González, Kulisevsky, Boltes, Otermín, \& García-Sánchez, 2003). Nonetheless, other studies found that the best index to discriminate between healthy aging and aMCI or AD (see the review of Carlesimo, Perri, \& Caltagirone, 2010) or to detect conversion from aMCI to AD (Sarazin et al., 2007) was immediate recall, supporting the encoding deficit hypothesis.

The encoding deficit hypothesis states that aMCI and AD patients are unable to correctly encode new verbal and visual material that they are learning (Corkin, 1982; Dick, Kean, \& Sands, 1989), and therefore they are unable to remember it later (see also Becker, Boller, Saxton, \& McGonigle-Gibson, 1987). The encoding deficit hypothesis is also observed despite controlling for initial learning (Christensen, Kopelman, Stanhope, Lorentz, \& Owen, 1998). Similar encoding deficits have also been reported in $\mathrm{AD}$ in both recall and recognition tasks (Martin, Brouwers, Cox, \& Fedio, 1985). The authors argued that AD patients are unable to encode a sufficient number of stimulus features or attributes, and therefore their memory traces are not rich enough to be retrieved later on.

Semantic features are known to provide important benefits to successful encoding in healthy 
older adults. In contrast, AD patients do not seem to benefit from deeper encoding (i.e. semantic processing) and exhibit a flat pattern of recognition across all conditions (Corkin, 1982; see also, Grober, Ocepek-Welikson, \& Teresi, 2009; Degenszajn, Caramelli, Caixeta, \& Nitrini, 2001; Tounsi et al., 1999). Furthermore, semantic cueing is not as useful in patients as in healthy older adults. However, correctly encoded items appear to remain preserved in memory since AD patients are able to correctly recall and recognize these items with the help of semantic cues (Oltra-Cucarella, Pérez-Elvira, \& Duque, 2014). Conversely, MCI patients benefit from semantic cueing, but only when cognitive support is provided (cued recall), although they show less semantic clustering than healthy adults (Ribeiro, Guerreiro, \& De Mendonça, 2007).

Taken together, these data do not allow us to clearly conclude in favor of either the encoding or the accelerated decline hypotheses in aMCI and AD. One could argue that a combination of encoding and consolidation deficits may account for the memory impairment observed in these conditions. In support of this view, several studies have reported a significant impairment of both encoding and consolidation in aMCI and AD (e.g., Delis et al., 1991; Reed, Paller, \& Mungas, 1998). For instance, Moulin et al. (Moulin, James, Freeman, \& Jones, 2004) compared the performance of aMCI and AD patients with that of controls on three different memory tasks. They found that patients exhibited both encoding and consolidation deficits, and these deficits were not correlated. The authors thus concluded that two different mechanisms were responsible for the memory deficits. According to another view (Ally et al., 2013), aMCI and AD are on a continuum. Patients with aMCI are able to correctly encode new information due to the mild nature of their memory impairment, but they suffer from a rapid decline of the memory trace. In contrast, with the progression of cognitive decline, AD patients suffer from both accelerated decline and impaired encoding.

According to the encoding deficit hypothesis, it can be hypothesized that once encoding has been controlled for, a similar rate of forgetting should be observed between groups, whereas the 
accelerated forgetting hypothesis predicts the opposite. However despite the different methods used to equate learning, some studies still found a more rapid decline of the memory trace despite attempts to control for encoding performance (Larrabee et al., 1993; Walsh et al., 2014), while others did not (Christensen et al., 1998; Kopelman, 1985).

The lack of consistency in these findings may be due to methodological discrepancies across studies. Nevertheless, effects predicted by each hypothesis were found regardless of the population (aMCI vs. AD), the task (recognition vs. free recall), and the material (verbal vs. visual). Surprisingly, almost all the studies reported above appeared to use an explicit learning phase ${ }^{1}$. Therefore, it is difficult to exclude the possible contribution of executive functioning in memory performance. It is well known that executive functions are involved in episodic encoding and recall in healthy young adults, healthy older adults (Troyer, Graves, \& Cullum, 1994), and individuals with aMCI or AD (e.g., Bisiacchi, Borella, Bergamaschi, Carretti, \& Mondini, 2008). Furthermore, to our knowledge, no study has manipulated inherent properties of items to be learned as based on the dual coding theory (Paivio, 1990). This theory posits that pictures of common objects benefit from dual coding involving their visual features and verbal labels, whereas abstract concepts and abstract pictures are considered to be encoded only on their visual features. Abstract items should thus be considered as weaker, and they should lead to a poorer encoding than common objects.

The present study aims to confront the encoding and accelerated forgetting hypotheses in aMCI and AD by manipulating (a) the delay in an incidental memory task; and (b) the nature of the items. Memory performance of healthy elderly (HE), aMCI, and AD patients was assessed using the DMS- 48 (Delayed-Matching-to Sample 48 items, Barbeau et al., 2004) in order to specifically investigate the respective roles of encoding versus forgetting memory processes. We chose the DMS- 48 because it is

1 It was not always specified whether or not the learning phase was explicit or incidental. 
commonly used in clinical practice, it offers an incidental learning phase followed by three forcedchoice visual recognition tests including a one-week delay, and it also distinguishes three different types of items in the recognition phases. Unique items include a picture of a real object (the target, e.g., a tree) presented along with an unrelated distractor (e.g., a book); Paired items include a real object (the target) presented along with another exemplar of the same category (e.g., two different models of cars). Finally, abstract items include both an abstract target and an abstract distractor (meaningless visual shapes, difficult to verbalize).

According to the rapid forgetting hypothesis, the most important decline in performance in the aMCI and AD groups should be found between the three-minute and one-hour delays when compared to the HE group. However, a decline in performance should also be noted, but to a lesser extent, between the one-hour and the one-week delays. The decline rates should be the same regardless of the type of items (unique, paired, or abstract). According to the encoding deficit hypothesis, aMCI and AD patients should present with the greatest deficits after the three-minute delay (irrespective of item type), whereas a relatively similar decline across aMCI, AD, and $\mathrm{HE}$ groups should be observed at the subsequent one-hour and one-week delays. Moreover, dual-coded items (visual + semantic: unique and paired items) should be better recognized than single-coded items (abstract items), because dual-coded items are assumed to have been encoded more profoundly, according to Paivio's (1990) dual-coding theory.

\section{Methods}

The present study was approved by the Ethics committee of the Research Center of Institut universitaire de gériatrie de Montréal and complies with the recognized standards on human experimentation (Helsinki Declaration of 1975, 2000). All participants signed an informed consent form before the start of the experimental session. A one-week delay separated the two sessions. They 
received a monetary compensation $(\$ 40)$ for their participation.

\section{Participants}

A total of 53 participants were recruited. Five participants with AD were excluded due to the fact that they did not complete the one-week delay test of the DMS- $48^{2}$. Thus, 48 participants were included in the present study (see Table 1). These participants were divided into three groups: 16 healthy elderly (HE), 16 patients with an amnestic Mild Cognitive Impairment (aMCI), and 16 patients with Alzheimer's disease (AD). Diagnosis of aMCI and AD patients was confirmed during a consensus meeting. All aMCI patients received a diagnosis of probable aMCI according to the criteria proposed by Petersen (Albert et al., 2011; Petersen, 2004). All of them reported a subjective memory complaint and exhibited a mild episodic memory impairment -that is, more than 1.5 standard deviations below the norm on standardized memory tests. None of them reported a significant impact of their memory impairment on their activities of daily living. All AD patients received a diagnosis of probable Alzheimer's disease according to the Diagnostic and Statistical Manual of Mental Disorders-Fourth Edition (DSM -IV; American Psychiatric Association, 1994) and the National Institute of Neurological and Communicative Disorders and Stroke-Alzheimer's Disease and Related Disorders Association (NINCDS-ADRDA) criteria (McKhann et al., 1984). They were in the early to moderate stages of the

2 The five AD patients excluded did not differ from those who completed all of the memory subtests, in terms of age, education, global cognitive functioning (MMSE and MOCA) and scores on the first two sets of the DMS-48. 
disease as defined by a Mini-Mental State Examination (MMSE) score between 20 and 28. All aMCI and AD patients were recruited at the Notre-Dame Hospital and the Institut universitaire de gériatrie de Montréal. Participants in the HE group were recruited in the Institut universitaire de gériatrie de Montréal and by public announcements in the community. Participants in the HE group were matched for education and age with aMCI and AD patients (see Table 1). 
Table 1. Means (and standard deviations) of demographic and global cognitive functioning data for the healthy elderly (HE), amnestic MCI (aMCI) and patient with Alzheimer's disease (AD).

\begin{tabular}{|c|c|c|c|c|c|}
\hline & $\underset{(n=16)}{H E}$ & $\begin{array}{l}\text { aMCI } \\
(n=16)\end{array}$ & $\underset{(n=16)}{A D}$ & $p$ & Comparison \\
\hline \multicolumn{6}{|l|}{ Demographic } \\
\hline Age & $74.94(7.5)$ & $78.19(6.1)$ & $77.44(5.9)$ & 0.35 & $\mathrm{HE}=\mathrm{aMCI}=\mathrm{AD}$ \\
\hline $\operatorname{Sex}(M / F)$ & $4 / 12$ & $5 / 11$ & $5 / 11$ & NA & NA \\
\hline Education (years) & $14.88(3.9)$ & $14.94(4.1)$ & $12.22(4.1)$ & 0.11 & $\mathrm{HE}=\mathrm{aMCI}=\mathrm{AD}$ \\
\hline \multicolumn{6}{|l|}{ Global } \\
\hline MMSE & $28.94(1.1)$ & $28.31(1.5)$ & $24.81(2)$ & $<0.001 * * *$ & $\mathrm{HE}=\mathrm{aMCI}>\mathrm{AD}$ \\
\hline MoCA & $28.06(1.5)$ & $25.62(2.1)$ & $20.31(3.4)$ & $<0.001 * * *$ & $\mathrm{HE}>\mathrm{aMCI}>\mathrm{AD}$ \\
\hline
\end{tabular}

Note. Means; standard deviations in parentheses. $\mathrm{HE}=$ healthy elderly; aMCI $=$ amnestic MCI; AD = Alzheimer's disease; $\mathrm{M}=$ male; $\mathrm{F}$ = female; $\mathrm{MMSE}=$ Mini-Mental State Examination; MoCA = Montréal Cognitive Assessment; NA = analysis not computed. $p=p$ values computed for each analysis of variance (ANOVA) with the group (HE, aMCI, AD) as a between -subjects variable. $* p<.05 . * * p<.01 . * * * p<.001$.

Health information was gathered from all participants during an extensive medical history and neuropsychology interview. Participants with a medical history and/or taking medications for conditions with known sensory or neurological effects were excluded. Participants who reported a diagnosis of depression or anxiety were included only if they were stable on their medication and if they were nonsymptomatic at the time of the study. All participants in the study were native French speakers and demonstrated adequate speech, visual, and hearing performances. Furthermore, all participants underwent a comprehensive neuropsychological assessment which was used to assess for the inclusion and exclusion criteria.

\section{Materials and procedure}

We chose the DMS-48 because this test is based on clear hypotheses concerning which aspect of memory should be impaired first in AD (Barbeau, Tramoni, et al., 2004; reviewed in Didic et al., 2013). The DMS-48 is a two-alternative forced-choice visual recognition task based on the classic delayed matching-to-sample task used in animals. The DMS-48 offers the following advantages: (a) There is an incidental learning phase -that is participants are unaware they are performing a memory test. Participants see a set of 48 colored drawings, which can represent objects, animals or abstract 
pictures. Yet, encoding is ensured by asking participants whether or not the picture is composed of three or more colors thus requiring a certain detail of perceptual analysis. Consequently, due to the incidental learning procedure, memory performance on this test should be not affected by executive functions or strategic encoding deficits; (b) after an interfering 2-min fluency task, participants complete an immediate (Set 1, 3 minutes later), followed by a delayed (Set 2,60 min later) and a long delayed (Set 3, 1 week later) forced-choice recognition task in which two drawings are presented at a time. Thus participants do not rely on effortful retrieval processes during the recognition phases; (c) this test is one of the very few developed tests that allows us to assess memory after a relatively long delay (i.e., one week-delay), a notable advantage over other tests. (d) The DMS-48 also enables to examine memory performance for three different types of items: unique (tree vs. book), paired (two different models of cars), and abstract items (meaningless visual shapes, difficult to verbalize). Unique items do not share semantic and perceptual features (color, shape, etc.) as opposed to paired items. Some of the abstract distractors also share visual features with their associated target (see examples in Appendix).

The following measures were recorded for each participant at each delay: Total - number of correct items recognized across all conditions; Unique - number of correct unique items recognized; Paired - number of correct paired items recognized and Abstract - number of correct abstract items recognized.

\section{Statistical Analyses}

Analyses of variance (ANOVAs) were conducted on the Total scores (sum of correct recognition across conditions) with the Group (HE vs. aMCI vs. $\mathrm{AD}$ ) as between-subjects variable and the Time (immediate vs. 1 hour vs. 1 week) as within-subjects variable. An item type ANOVA was also conducted on the scores of each condition with the Group (HE vs. aMCI vs. AD) as between- 
subjects variable and the Time (immediate vs. 1 hour vs. 1 week) and the Item type (unique vs. paired vs. abstract) as within-subjects variables. Mauchly's test for sphericity was conducted for each ANOVA to assess the homogeneity of variance, and the analyses did not reveal any significant effect. Therefore, the ANOVAs were not corrected. Post hoc analyses were computed using unilateral Student's $t$-test corrected for multiple comparisons using the Holmes procedure. A $p$ value of $<.05$ was used as significant threshold for all the analyses. One AD patient did not complete the last set of the DMS-48 (set 3). Thus, resulting missing values were replaced by the different means of the group of $\mathrm{AD}$ patients in the different conditions (total, abstract, paired, unique).

\section{Results}

\section{Total Scores}

The ANOVA performed on the Total scores of the DMS-48 (see Figure 1) revealed a main effect of the Group, $F(2,45)=37.79, p<.05, \eta^{2} g=.56 . \mathrm{HE}(M=46, S D=2)$ performed better than aMCI patients $(M=45, S D=2.9)$ who performed better than AD patients $(M=36, S D=6.2$; all $p<.05)$. The Time factor was also significant, $F(2,90)=5.24, p<.05, \eta^{2} g=.03$. A significant difference was observed between immediate recognition and one-week delay $(p<.05)$, but there was no difference between immediate recognition and one-hour delay $(p=.1)$, nor was there a difference between the one-hour and one-week delay $(p=.1)$. Finally, the Group by Time interaction was not significant, $F(4,90)<1$. 


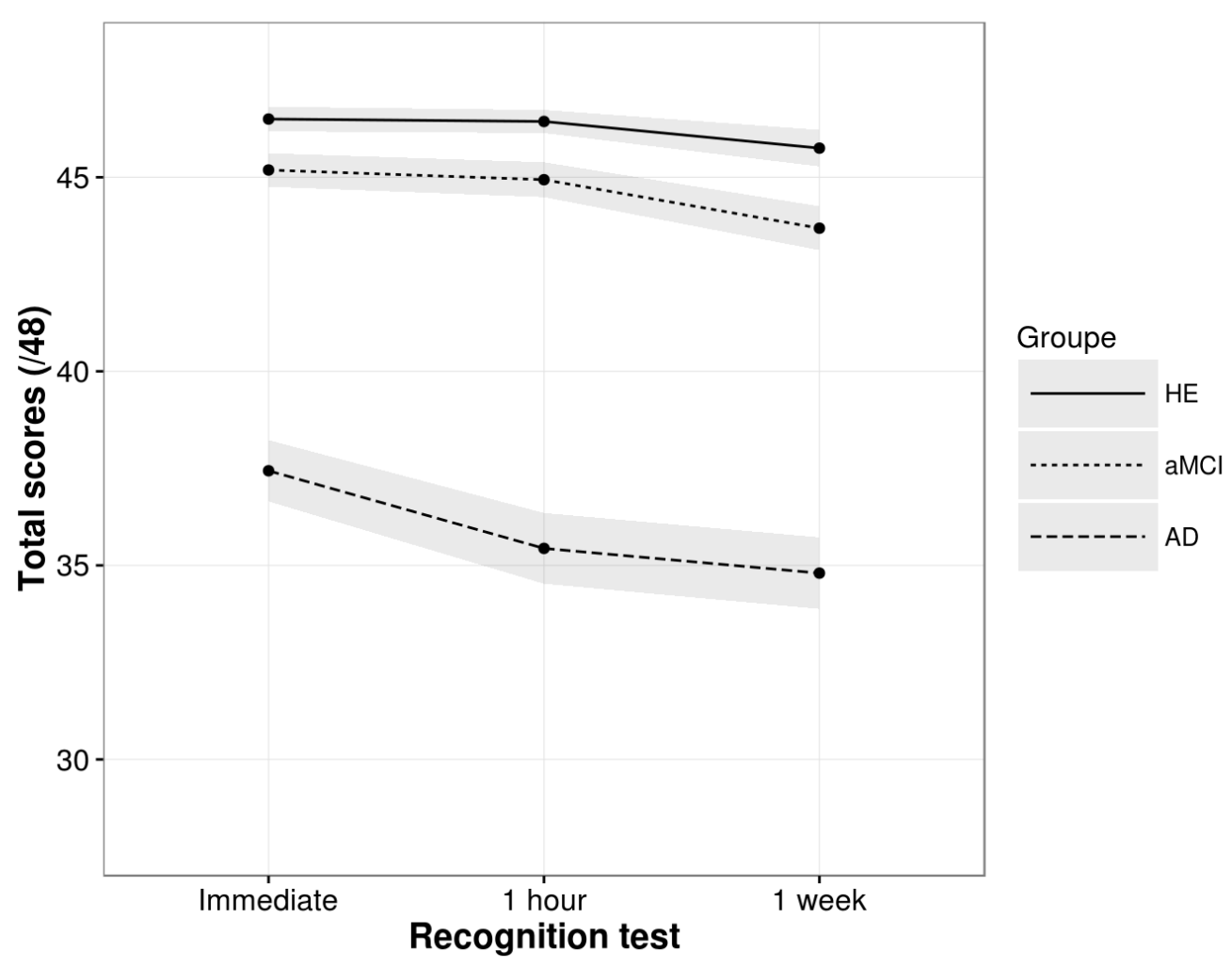

Figure 1. Total correct recognition hits for healthy control (HE), amnestic MCI patients (aMCI), and patients with Alzheimer's disease (AD) at different delays. Grey ribbons represent standard errors corrected for within-subjects design.

\section{Item-type}

Beyond the effect of Group and Time factors (see the Total Scores subsection), the item-type analysis revealed a significant effect of the Item-type factor, $F(2,90)=22.46, p<.05, \eta^{2} g=.08$ with the Unique items better recognized than the Paired and Abstract items $(p<.05)$ which were marginally different one from each other $(p=.06)$.

The Group by Item-Type interaction $(F(4,90)<1)$ as well as the double interaction Group by Time by Item-Type $\left(F(4,180)=3.16, p<.05, \eta^{2} g=.02\right)$ were significant. 
Figure 2). The ANOVA performed in the HE group showed only a significant effect of the Item-Type factor, $F(2,30)=3.47, p<.05, \eta^{2} g=.06$, whereas the Time factor $\left(F(2,30)=1.26, p=.30, \eta^{2} g=.01\right)$ and the Time by Item-type interaction $\left(F(4,60)=1.32, p=.27, \eta^{2} g=.02\right)$ were not significant. Unique items were better recognized than paired and abstract items $(p<.05)$, but no difference was observed between paired and abstract items $(p=.23)$.

The ANOVA performed with the aMCI group revealed the same pattern of results as that in the HE group. The only significant effect was observed for the Item-Type factor, $F(2,30)=6.56, p<.05$, $\eta^{2} g=.08$, whereas a trend was observed for the Time factor $\left(F(2,30)=2.74, p=.08, \eta^{2} g=.03\right)$ and no effect for the Time by Item-type interaction $\left(F(4,60)=1.06, p=.38, \eta^{2} g=.02\right)$. Unique items were better recognized than paired and abstract items $(p<.05)$, but no difference was observed between paired and abstract items $(p=.20)$.

Finally, the ANOVA performed in the AD group revealed a significant effect of the Item-type factor, $F(2,30)=13.33, p<.05, \eta^{2} g=.11$ and of the Time by Item-Type interaction, $F(4,60)=3.70$, $p<.05, \eta^{2} g=.05$. A trend was observed for the Time factor, $F(2,30)=2.47, p=.10, \eta^{2} g=.02$. In the immediate recognition test, the unique and paired items $(p=.47)$ were better recognized than abstract items $(p<.05)$, whereas Unique items were better recognized than paired and abstract items in both the one-hour and the one-week later tests. In these recognition test times, paired and abstract items were not differently recognized $(p=.28, p=.37$ respectively). 


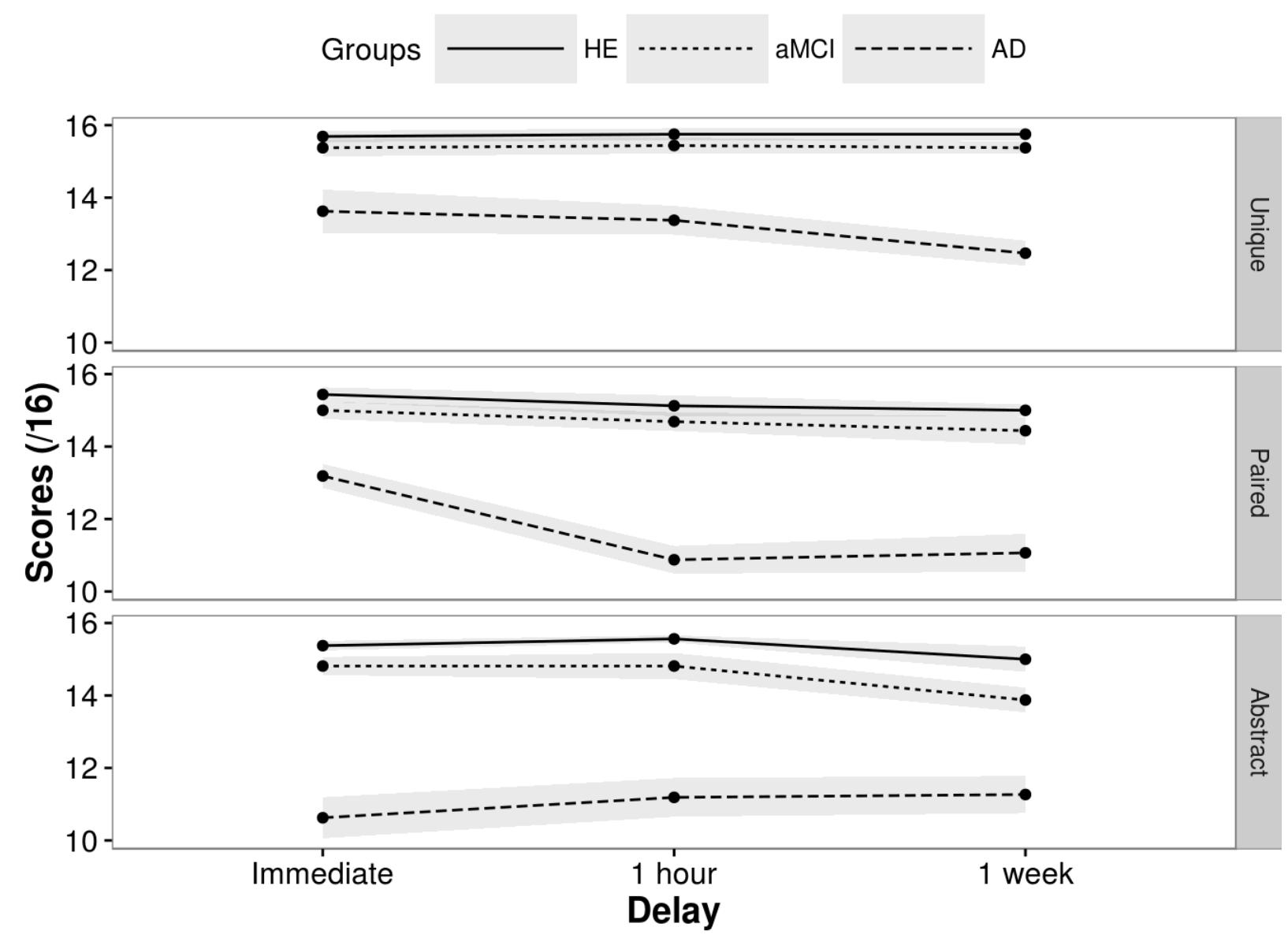

Figure 2. Number of hits per item type for healthy control (HE), amnestic MCI patients (aMCI), and patients with Alzheimer's disease (AD) at the three delays (immediate, 1 hour, 1 week). Grey ribbons represent standard errors

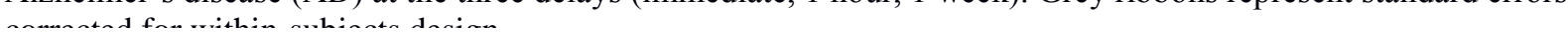

Figure 2 suggests a possible decline in performance in aMCI for abstract items at the one-week delay. As this observation may be consistent with the statistical analyses reported in the AD group, we conducted an exploratory analysis on the item type in aMCI group at the one-week delay, despite the fact that the Recognition Time by Item Type factor was not significant in this group. Pairwise $t$ tests indicated that fewer abstract items were recognized than unique items $(p<.05)$ supporting the descriptive observation. Recognition of abstract and paired items did not differ significantly $(p=.13)$, but marginally fewer paired items than unique items were recognized $(p=.08)$. 


\section{Discussion}

The present study aimed to test the accelerated forgetting and the encoding deficit hypotheses of memory deficits in aMCI and AD. We used the DMS-48 test in order to compare recognition performance of $\mathrm{HE}$, aMCI, and $\mathrm{AD}$ patients at immediate recognition, one-hour, and one-week delays. Contrary to most of the clinical tests used to assess episodic memory, the DMS-48 provides an incidental learning phase followed by three recognition phases. Therefore, by limiting the contribution of executive processes, it can be considered to assess "purer" memory processes. Another advantage of this test is that it allows us to compare recognition of three different item types: unique, paired and abstract. We discuss the results in light of both hypotheses. Since the data are in partial agreement with both of these two hypotheses, we propose a third possible interpretation based on the memory strength theory inspired from studies in normal cognition.

On the one hand, the rapid decay hypothesis assumes that recognition of all three item types rapidly declines over time in aMCI and AD compared to controls. It could also be assumed that the decrement would be more important between immediate and one-hour delays than between one-hour and one-week delays. Nonetheless, we did not observe a significant decline in performance between immediate recognition and one-hour delays. Recognition rates, however, were significantly poorer after one-week delay than at immediate recognition in all groups. This more pronounced drop in performance after a one-week delay has been recently reported by Walsh et al. (2014) in people with aMCI. A more rapid decline has been reported in AD (see, for instance, Carlesimo et al., 1993; Hart et al., 1988). Contrary to what would be predicted by the rapid decay hypothesis, the decline over time in the current study was, however, similar in patients and HE. The difference in findings between the present study and other studies reported in the literature may result from the intrinsic properties of the DMS-48. Finally, when looking more specifically at the item types, not all types declined over time, as would have been predicted by the rapid decay hypothesis. Abstract items were the most poorly 
recognized items, but their level of recognition remained almost identical across all delays in the three groups. This difficulty cannot be explained by a deficit in semantic elaboration in aMCI or AD since (a) they are known to benefit from the depth of encoding for pictures (picture superiority effect, Ally, Gold, \& Budson, 2009), and (b) HE also showed a similar effect (but see Joubert et al., 2010).

On the other hand, the encoding hypothesis would predict deficits in aMCI and $\mathrm{AD}$ in immediate recognition, while a decline rate similar to controls would be predicted at subsequent delays. As reported above, performance declined similarly in all groups between immediate recognition and the one-week delay, but not between immediate and one-hour delays. Yet, as predicted by the encoding hypothesis, abstract items were more poorly recognized than unique and paired items in $\mathrm{AD}$. This particular difficulty can be interpreted as resulting from the "dual coding" (Paivio, 1990) of unique and paired items (visual features + semantic label) relative to the single coding of abstract items (visual features only). Abstract items may thus not gain from deeper encoding and/or semantic elaboration (Craik \& Tulving, 1975), as opposed to paired and unique items. They may therefore be more difficult to recognize. Nonetheless, the difference in immediate recognition performance between unique and paired items in $\mathrm{AD}$ would not be predicted by the encoding hypothesis, since unique and paired items should be expected to benefit equally from dual coding.

The fact that unique items were better recognized than paired items may thus depend on task difficulty. It is indeed harder to distinguish among previously seen items between two very similar than two very distinctive items (e.g., Koutstaal \& Schacter, 1997). It has been shown that AD patients are able to benefit from conceptual and visual distinctive features in memory (Deason, Hussey, Flannery, \& Ally, 2015). Therefore, one can imagine that the worse performance of aMCI and AD patients than of HE comes from a degradation in the quality of memory traces. According to the fuzzy-trace theory (Brainerd \& Reyna, 2002), the verbatim process allows one to retrieve a memory as a whole with all its associated details, whereas the gist process refers to more generic and less detailed features. Previous 
data have shown that AD patients, and to some extend aMCI patients (Hudon et al., 2006), exhibit a verbatim impairment and thus rely to a greater extent upon gist memory (e.g., Budson, Todman, \& Schacter, 2006). Therefore, these patients exhibit worst performance in recall and correct recognition of items, especially when these items are presented with similar distractors. Memory performance of aMCI and AD patients in the present study could thus be explained by an encoding deficit combined with a greater reliance on gist memory.

Nonetheless, an interpretation based on these two hypotheses seems insufficient to account for the main finding of the present study regarding the pattern of recognition impairment in AD over time as function of item type. In $\mathrm{AD}$, abstract items were more poorly recognized from the beginning (immediate recognition) and the degree of recognition of abstract items remained the same at subsequent delays. Paired and unique items were more poorly recognized in $\mathrm{AD}$ than in aMCI and HE groups during immediate recognition, but were significantly better processed than abstract items in the AD group. At the one-hour delay, recognition of paired items declined at the same rate of recognition of abstract items in $\mathrm{AD}$, whereas recognition rates of unique items remained the same both at immediate and at one-hour delays. Finally, recognition of unique items declined after one week in AD patients, although these items remained better recognized at this delay than abstract and paired items.

The progressive decline in recognition memory over time as a function of item type evokes a loss of the strength or distinctiveness of the memory traces. Weaker traces should decline more rapidly over time than stronger traces and should also be less distinctive. Abstract items are particularly vulnerable since the traces created during their encoding are shallower - that is, weaker- than unique and paired items due the single encoding of the item (only visual compared to both visual and semantic features). This strength-based hypothesis (see Rohrer, 1996 for data in normal cognition) may also account for the progressive decline of paired and then unique items in recognition memory performance in $\mathrm{AD}$. Compared to $\mathrm{HE}$, the weaker memory traces in $\mathrm{AD}$ make them (a) decline more 
rapidly over time, which explains the difference between the groups, and (b) less distinctive, which explains why paired items decline before unique items. This idea of memory trace weakening was put forward in previous studies to account for amnesia. For instance, memory performance of amnesic patients may be reproduced in healthy young and/or elderly adults by increasing the delay of testing (Van der Linden \& Bruyer, 1991) or by degrading the perceptual information available (HE, aMCI and AD patients, Cronin-Golomb, Gilmore, Neargarder, Morrison, \& Laudate, 2007). These effects were interpreted by the authors based on previous findings that amnesic patients have weaker memory traces than controls (see also Berry, Kessels, Wester, \& Shanks, 2014). Alternatively, weaker memory traces may decline at a similar rate than stronger traces. The difference in performance may thus result from the activation/strength needed to pass a certain threshold below which a memory trace may not be accurately retrieved (see activation models of memory, such as MINERVA, Hintzman, 1984).

One implication of the strength-based hypothesis is that it gives a more central role of the sensorimotor components in the distinctiveness, and therefore in the strength, of the memory traces as demonstrated in young and healthy elderly adults (e.g., Brunel, Goldstone, Vallet, Riou, \& Versace, 2013; Vallet, Simard, Versace, \& Mazza, 2013). This role, combined with the disconnection syndrome reported in AD (Delbeuck, van der Linden, \& Collette, 2003), might also account for some of the memory disorders in AD (see Vallet, Hudon, Simard, \& Versace, 2013). Therefore, one may envision improving memory stimulation programs by focusing on sensorimotor components and their integration into the memory trace (e.g., Maseda, Sánchez, Marante, González-Abraldes, Buján, \& Millán-Calenti, 2014).

The memory strength hypothesis is partially supported in aMCI as indicated by the exploratory analysis in the present study. Abstract items started to significantly decline in the one-week delay whereas unique items remained the best recognized item type. In other words, this pattern suggests that abstract items may be the most vulnerable to decline in aMCI, but this effect may take more than a one- 
week delay to become clinically relevant and significant (at least in our group of aMCI patients). This finding may appear surprising since one might expect the performance of aMCI patients to stand midway between that of $\mathrm{HE}$ and $\mathrm{AD}$ patients. We found that $\mathrm{AD}$ patients exhibited the worst performance followed by the aMCI patients and then the HE as previously reported with DMS-48 test (Barbeau, Didic, et al., 2004; Didic et al., 2013). Nonetheless, in the current study the performance of the aMCI group was much similar to that of the HE group. This differs from previous studies and may be due to the fact that our aMCI group appeared to be globally less cognitively impaired than in other studies, such as indicated by the mean MMSE scores, and this despite the fact that our group was older (MMSE: 28.3 vs.27.1 vs. 26.6; Age: 78.1 vs. 71.8 vs 71.0 in the present study, in Didic et al., 2013 and Barbeau et al., 2004b respectively). Thus, more cognitively impaired aMCI patients may present lower scores for the abstract items. Future studies could address this issue by taking into account the itemtype factor into the analyses of performance of aMCI patients in the DMS-48 test. Further studies should also attempt to determine whether global processing of objects such as the stimuli used in the current study may be affected in AD and whether this may have an impact on performance. Finally, the modest sample size of the groups may reduce the statistical power of the study and results should therefore be interpreted accordingly.

One point that deserves mention is that the items in the DMS-48 test are context-free, as opposed to context-rich items in other memory tests (e.g., Family pictures subtests from the Wechsler Memory Scale; Wechsler, 1997). In fact, context-free recognition is presumed to rely on the perirhinal cortex, and this structure is indeed believed to be the first to be affected in AD (Barbeau, Sonetheiner, et al., 2004; Barbeau, Tramoni, et al., 2004; Braak \& Braak, 1991). Therefore, tests that rely upon context-free memory may detect the first detectable sign of memory impairment in AD (Didic et al. 2011). Once again, it is worth mentioning that the DMS-48 is unique in that it allows us to assess episodic memory using an incidental learning phase combined with a simple forced-choice recognition 
task. Most of the episodic tests commonly used in clinical practice rely on explicit learning and on a combination of free-recall and/or recognition tasks.

The present study is among the few testing memory performance at a one-week delay, and it is the first to follow an item-type approach to analyze the data of the DMS-48. This approach allowed us to find a very specific pattern of recognition impairment in $\mathrm{AD}$, with worst performance for abstract items at immediate delays followed by paired items at a one-hour delay and unique items at a one-week delay. This pattern of memory impairment in $\mathrm{AD}$ is neither compatible with the rapid decay hypothesis nor compatible with the encoding deficit hypothesis nor even with a combination of these two hypotheses. We proposed an alternative interpretation based on the strength of the memory trace taking into account both encoding and retrieval processes over time. However, our results are limited to recognition memory. Future studies are thus needed to test whether these results can be extended to free-recall paradigms. To summarize, the strengths of the present study are that: (a) the cognitive load is reduced by proposing an incidental learning phase and forced-choice recognition tasks; (b) a more qualitative approach is followed by considering an item-type analysis; (c) we use a clinical memory test designed to assess memory performance up to a one-week delay; (d) we contrast performance of patients with mild-cognitive impairment and patients with $\mathrm{AD}$ with healthy elderly adults. However, the present study is limited by (a) the particularities of the DMS-48 compared to more commonly used memory tests (incidental, context-free, recognition only); (b) the MCI patients being only mildly impaired; (c) the limited sample size. Considering the particularity of the DMS-48, our findings need to be replicated using more commonly used tests of episodic memory to further explore and understand the strength-based hypothesis. 


\section{Acknowledgements}

Guillaume Vallet is supported by a Fonds de Recherche du Québec en Santé (FRQ-S)

postdoctoral award. Sven Joubert and Isabelle Rouleau are supported by the Alzheimer Society of Canada. Sven Joubert is supported by a chercheur-boursier senior FRQ-S award. 


\section{References}

Albert, M. S., DeKosky, S. T., Dickson, D., Dubois, B., Feldman, H. H., Fox, N. C., Gamst, A., et al. (2011). The diagnosis of mild cognitive impairment due to Alzheimer's disease:

Recommendations from the National Institute on Aging-Alzheimer's Association workgroups on diagnostic guidelines for Alzheimer's disease. Alzheimer's \& Dementia, 7, 270-279. doi:10.1016/j.jalz.2011.03.008

Ally, B. A., Gold, C. A., \& Budson, A. E. (2009). The picture superiority effect in patients with Alzheimer's disease and mild cognitive impairment. Neuropsychologia, 47, 595-598. doi:10.1016/j.neuropsychologia.2008.10.010

Ally, B. A., Hussey, E. P., Ko, P. C., \& Molitor, R. J. (2013). Pattern separation and pattern completion in Alzheimer's disease: Evidence of rapid forgetting in amnestic mild cognitive impairment. Hippocampus, 23, 1246-1258. doi:10.1002/hipo.22162

Barbeau, E., Tramoni, E., Joubert, S., Mancini, J., Ceccaldi, M., Poncet, M. (2004a). Evaluation de la mémoire de reconnaissance visuelle: normalisation d'une nouvelle épreuve en choix forcé (DMS48) et utilité en neuropsychologie clinique. L'évaluation des troubles de la mémoire. Marseille: Solal, 85-101.

Barbeau, E. J., Didic, M., Tramoni, E., Felician, O., Joubert, S., Sontheimer, A., Ceccaldi, M., et al. (2004b). Evaluation of visual recognition memory in MCI patients. Neurology, 62, 1317-1322.

Barbeau, E., Sontheimer, A., Joubert, S., Didic, M., Felician, O., Tramoni, E., et al.(2004c). [The human perirhinal cortex]. Revue neurologique, 160, 401-411.

Bäckman, L., Jones, S., Berger, A.-K., Laukka, E. J., \& Small, B. J. (2005). Cognitive impairment in preclinical Alzheimer's disease: A meta-analysis. Neuropsychology, 19, 520-531. doi:10.1037/0894-4105.19.4.520 
Becker, J. T., Boller, F., Saxton, J., \& McGonigle-Gibson, K. L. (1987). Normal rates of forgetting of verbal and non-verbal material in Alzheimer's disease. Cortex, 23, 59-72. doi:10.1016/S0010-9452(87)80019-9

Bennett, I. J., Golob, E. J., Parker, E. S., \& Starr, A. (2006). Memory evaluation in mild cognitive impairment using recall and recognition tests. Journal of Clinical and Experimental Neuropsychology, 28, 1408-1422. doi:10.1080/13803390500409583

Benton, A. L., Hamsher, K. D., Varney, N. R., \& Spreen, O. (1983). Judgment of line orientation. Oxford University Press New York.

Berry, C. J., Kessels, R. P. C., Wester, A. J., \& Shanks, D. R. (2014). A single-system model predicts recognition memory and repetition priming in amnesia. The Journal of Neuroscience: The Official Journal of the Society for Neuroscience, 34, 10963-10974. doi:10.1523/JNEUROSCI.076414.2014

Bird, M., \& Luszcz, M. (1991). Encoding specificity, depth of processing, and cued recall in Alzheimer's disease. Journal of Clinical and Experimental Neuropsychology, 13, 508-520. doi:10.1080/01688639108401067

Bisiacchi, P. S., Borella, E., Bergamaschi, S., Carretti, B., \& Mondini, S. (2008). Interplay between memory and executive functions in normal and pathological aging. Journal of Clinical and Experimental Neuropsychology, 30, 723-733. doi:10.1080/13803390701689587

Brainerd, C. J., \& Reyna, V. F. (2002). Fuzzy-trace theory and false memory. Current Directions in Psychological Science, 11, 164-169. doi: 10.1111/1467-8721.00192

Brunel. L., Goldstone, R. L., Vallet, G. T., Riou, B., \& Versace, R. (2013). When seing a dog activates the bark: Multisensory generalization and distinctiveness effects. Experimental Psychology, 
60, 100-112. doi: 10.1027/1618-3169/a000176.

Budson, A. E., Simons, J. S., Sullivan, A. L., Beier, J. S., Solomon, P. R., Scinto, L. F., et al. (2004). Memory and emotions for the September 11, 2001, terrorist attacks in patients with Alzheimer's disease, patients with mild cognitive impairment, and healthy older adults. Neuropsychology, 18, 315-327. doi:10.1037/0894-4105.18.2.315

Budson, A. E., Simons, J. S., Waring, J. D., Sullivan, A. L., Hussoin, T., \& Schacter, D. L. (2007). Memory for the September 11, 2001, terrorist attacks one year later in patients with Alzheimer's disease, patients with mild cognitive impairment, and healthy older adults. Cortex, 43 , 875-888.

Budson, A. E., Todman, R. W., \& Schacter, D. L. (2006). Gist memory in Alzheimer's disease: Evidence from categorized pictures. Neuropsychology, 20, 113-122. doi:10.1037/0894-4105.20.1.113

Carlesimo, G. A., \& Oscar-Berman, M. (1992). Memory deficits in Alzheimer's patients: A comprehensive review. Neuropsychology Review, 3, 119-169.

Carlesimo, G. A., Fadda, L., Bonci, A., \& Caltagirone, C. (1993). Differential rates of forgetting from long-term memory in Alzheimer's and multi-infarct dementia. The International Journal of Neuroscience, 73.

Carlesimo, G. A., Perri, R., \& Caltagirone, C. (2010). Category cued recall following controlled encoding as a neuropsychological tool in the diagnosis of Alzheimer's disease: A review of the evidence. Neuropsychology Review, 21, 54-65. doi:10.1007/s11065-010-9153-7

Christensen, H., Kopelman, M. D., Stanhope, N., Lorentz, L., \& Owen, P. (1998). Rates of forgetting in Alzheimer dementia. Neuropsychologia, 36(6), 547-557. doi:10.1016/S0028- 
Corkin, S. (1982). Some relationships between global amnesias and the memory impairments in Alzheimer's disease. In S. Corkin, K. L. Davis, J. H. Growdon, E. Usdin, \& R. Wurtman (Eds.), Alzheimer's disease: A report of progress in research (Raven Pres., pp. 149-164). New York: Raven Press.

Craik, F. I. M., \& Tulving, E. (1975). Depth of processing and the retention of words in episodic memory. Journal of Experimental Psychology: General, 104, 268-294. doi:10.1037//00963445.104.3.268

Cronin-Golomb, A., Gilmore, G., Neargarder, S., Morrison, S., \& Laudate, T. (2007). Enhanced stimulus strength improves visual cognition in aging and Alzheimer's disease. Cortex, 43, 952-966.

Crowell, T. A., Luis, C. A., Vanderploeg, R. D., Schinka, J. A., \& Mullan, M. (2002). Memory patterns and executive functioning in mild cognitive impairment and Alzheimer's disease. Aging Neuropsychology and Cognition, 9, 288-297. doi:10.1076/anec.9.4.288.8772

Deason, R. G., Hussey, E. P., Flannery, S., \& Ally, B. A. (2015). Preserved conceptual implicit memory for pictures in patients with Alzheimer's disease. Brain and Cognition, 99, 112-117. doi:10.1016/j.bandc.2015.07.008

Degenszajn, J., Caramelli, P., Caixeta, L., \& Nitrini, R. (2001). Encoding process in delayed recall impairment and rate of forgetting in Alzheimer's disease. Arquivos de Neuro-Psiquiatria, 59, 171-174. doi:10.1590/S0004-282X2001000200003

Delbeuck, X., Van der Linden, M., \& Collette, F. (2003). Alzheimer's Disease as a Disconnection Syndrome? Neuropsychology Review, 13, 79-92. doi:10.1023/A:1023832305702

Delis, D. C., Massman, P. J., Butters, N., Salmon, D. P., Cermak, L. S., \& Kramer, J. H. (1991). Profiles of demented and amnesic patients on the California Verbal Learning Test: Implications for the 
assessment of memory disorders. Psychological Assessment: A Journal of Consulting and Clinical Psychology, 3, 19-26. doi:10.1037/1040-3590.3.1.19

Dick, M. B., Kean, M.-L., \& Sands, D. (1989). Memory for action events in Alzheimer-type dementia: Further evidence of an encoding failure. Brain and Cognition, 9, 71-87. doi:10.1016/02782626(89)90045-6

Didic, M., Barbeau, E. J., Felician, O., Tramoni, E., Guedj, E., Poncet, M., \& Ceccaldi, M. (2011). Which memory system is impaired first in Alzheimer's disease? Journal of Alzheimer's Disease, 27, 11-22. doi:10.3233/JAD-2011-110557

Didic, M., Felician, O., Barbeau, E. J., Mancini, J., Latger-Florence, C., Tramoni, E., \& Ceccaldi, M. (2013). Impaired visual recognition memory predicts Alzheimer's disease in amnestic mild cognitive impairment. Dementia and Geriatric Cognitive Disorders, 35, 291-299. doi:10.1159/000347203

Estévez- González, A., Kulisevsky, J., Boltes, A., Otermín, P., \& García-Sánchez, C. (2003). Rey verbal learning test is a useful tool for differential diagnosis in the preclinical phase of Alzheimer's disease: Comparison with mild cognitive impairment and normal. International Journal of Geriatric Psychiatry, 18, 1021-1028.

Fleischman, D. A., \& Gabrieli, J. D. E. (1999). Long-term memory in Alzheimer's disease. Current Opinion in Neurobiology, 9, 240-244.

Folstein, M. F., Folstein, S. E., \& McHugh, P. R. (1975). “Mini-mental state”: A practical method for grading the cognitive state of patients for the clinician. Journal of Psychiatric Research, 12, 189-198. doi:10.1016/0022-3956(75)90026-6

Greenaway, M. C., Lacritz, L. H., Binegar, D., Weiner, M. F., Lipton, A., \& Munro Cullum, C. 
(2006). Patterns of verbal memory performance in mild cognitive impairment, Alzheimer disease, and normal aging. Cognitive and Behavioral Neurology, 19, 79-84.

doi:10.1097/01.wnn.0000208290.57370.a3

Grober, E., Ocepek-Welikson, K., \& Teresi, J. A. (2009). The free and cued selective reminding test: Evidence of psychometric adequacy. Psychology Science Quarterly, 51, 266-282.

Hart, R. P., Kwentus, J. A., Harkins, S. W., \& Taylor, J. R. (1988). Rate of forgetting in mild Alzheimer's-type dementia. Brain and Cognition, 7, 31-38. doi:10.1016/0278-2626(88)90019-X

Hintzman, D. L. (1984). MINERVA 2: A simulation model of human memory. Behavior Research Methods, Instruments, \& Computers, 16, 96-101.

Hudon, C., Belleville, S., Souchay, C., Gély-Nargeot, M.-C., Chertkow, H., \& Gauthier, S. (2006). Memory for gist and detail information in Alzheimer's disease and mild cognitive impairment. Neuropsychology, 20, 566-577.

Joubert, S., Brambati, S. M., Ansado, J., Barbeau, E. J., Felician, O., Didic, M., ... Kergoat, M.J. (2010). The cognitive and neural expression of semantic memory impairment in mild cognitive impairment and early Alzheimer's disease. Neuropsychologia, 48, 978-988. doi:10.1016/j.neuropsychologia.2009.11.019

Kopelman, M. D. (1985). Rates of forgetting in Alzheimer-type dementia and Korsakoff's syndrome. Neuropsychologia, 23, 623-638. doi:10.1016/0028-3932(85)90064-8

Koutstaal, W., \& Schacter, D. L. (1997). Gist-based false recognition of pictures in older and younger adults. Journal of Memory and Language, 37, 555-583.

Larrabee, G. J., Youngjohn, J. R., Sudilovsky, A., \& Crook, T. H. (1993). Accelerated 
forgetting in Alzheimer-type dementia. Journal of Clinical and Experimental Neuropsychology, 15, 701-712. doi:10.1080/01688639308402590

Manes, F., Serrano, C., Calcagno, M. L., Cardozo, J., \& Hodges, J. (2008). Accelerated forgetting in subjects with memory complaints. A new form of Mild Cognitive Impairment? Journal of Neurology, 255, 1067-1070. doi:10.1007/s00415-008-0850-6

Martin, A., Brouwers, P., Cox, C., \& Fedio, P. (1985). On the nature of the verbal memory deficit in Alzheimer's disease. Brain and Language, 25, 323-341.

Maseda, A., Sánchez, A., Marante, M. P., González-Abraldes, I., Buján, A., \& Millán-Calenti, J. C. (2014). Effects of multisensory stimulation on a sample of institutionalized elderly people with dementia diagnosis: A controlled longitudinal trial. American Journal of Alzheimer's Disease and Other Dementias, 29, 463-473. doi:10.1177/1533317514522540

McKhann, G., Drachman, D., Folstein, M., Katzman, R., Price, D., \& Stadlan, E. M. (1984). Clinical diagnosis of Alzheimer's disease: Report of the NINCDS-ADRDA Work Group under the auspices of Department of Health and Human Services Task Force on Alzheimer's Disease. Neurology, 34, 939-944.

Moulin, C. J., James, N., Freeman, J. E., \& Jones, R. W. (2004). Deficient acquisition and consolidation: Intertrial free recall performance in Alzheimer's disease and Mild Cognitive Impairment. Journal of Clinical and Experimental Neuropsychology, 26, 1-10. doi:10.1076/jcen.26.1.1.23940

Oltra-Cucarella, J., Pérez-Elvira, R., \& Duque, P. (2014). Benefits of deep encoding in Alzheimer disease. Analysis of performance on a memory task using the item specific deficit approach. Neurologia, 29, 286-293. doi:10.1016/j.nrl.2013.06.006 
Paivio, A. (1990). Mental representations: A dual coding approach. Oxford: Oxford University Press.

Petersen, R. C. (2004). Mild cognitive impairment as a diagnostic entity. Journal of Internal Medicine, 256, 183-194. doi:10.1111/j.1365-2796.2004.01388.x

Psychiatric, A. (1994). No Diagnostic and Statistical Manual of Mental Disorders, 4th edition. Washington, DC.

Reed, B. R., Paller, K. A., \& Mungas, D. (1998). Impaired acquisition and rapid forgetting of patterned visual stimuli in Alzheimer's disease. Journal of Clinical and Experimental Neuropsychology, 20, 738-749. doi:10.1076/jcen.20.5.738.1123

Ribeiro, F., Guerreiro, M., \& De Mendonça, A. (2007). Verbal learning and memory deficits in Mild Cognitive Impairment. Journal of Clinical and Experimental Neuropsychology, 29, 187-197. doi:10.1080/13803390600629775

Rohrer, D. (1996). On the relative and absolute strength of a memory trace. Memory \& Cognition, 24, 188-201.

Sarazin, M., Berr, C., De Rotrou, J., Fabrigoule, C., Pasquier, F., Legrain, S., et al. (2007). Amnestic syndrome of the medial temporal type identifies prodromal AD: A longitudinal study. Neurology, 69, 1859-1867. doi:10.1212/01.wnl.0000279336.36610.f7

Stopford, C. L., Snowden, J. S., Thompson, J. C., \& Neary, D. (2007). Distinct memory profiles in Alzheimer's disease. Cortex, 43, 846-857. doi:10.1016/S0010-9452(08)70684-1

Tounsi, H., Deweer, B., Ergis, A. M., Van der Linden, M., Pillon, B., Michon, A., \& Dubois, B. (1999). Sensitivity to semantic cuing: an index of episodic memory dysfunction in early Alzheimer 
disease. Alzheimer Disease and Associated Disorders, 13, 38-46.

Troyer, A. K., Graves, R. E., \& Cullum, C. M. (1994). Executive functioning as a mediator of the relationship between age and episodic memory in healthy aging. Aging, Neuropsychology and Cognition, 1, 45-53.

Tulving, E. (1983). Elements of Episodic Memory. Oxford: Clarendon Press.

Tulving, E. (2002). Episodic memory: from mind to brain. Annual Review of Psychology, 53, 125. doi:10.1146/annurev.psych.53.100901.135114

Vallet, G. T., Simard, M., Versace, R., \& Mazza, S. (2013). The perceptual nature of audiovisual interactions for semantic knowledge in young and elderly adults. Acta Psychologica, 143, 253-260. doi:10.1016/j.actpsy.2013.04.009

Vallet, G. T., Hudon, C., Simard, M., \& Versace, R. (2013). The disconnection syndrome in the Alzheimer's disease: The cross-modal priming example. Cortex, 49, 2402-2415. doi:10.1016/j.cortex.2012.10.010

Van der Linden, M., \& Bruyer, R. (1991). Neuropsychologie de la mémoire humaine. Grenoble: Presses Universitaires de Grenoble (PUG).

Walsh, C. M., Wilkins, S., Bettcher, B. M., Butler, C. R., Miller, B. L., \& Kramer, J. H. (2014). Memory consolidation in aging and MCI after 1 week. Neuropsychology, 28, 273-380. doi:10.1037/neu0000013

Welsh, K. A., Butters, N., Hughes, J. P., Mohs, R. C., \& Heyman, A. (1992). Detection and staging of dementia in Alzheimer's disease. Use of the neuropsychological measures developed for the Consortium to Establish a Registry for Alzheimer's Disease. Archives of Neurology, 49, 448-452. 


\section{Appendix}

Examples of stimuli used in the DMS-48 as a function of the item type (unique, paired, abstract) and of the recognition delay ( 3 min., 1 hour, 1 week). Note that items are in color in the original format.

\section{$3 \mathrm{~min}$.}

\section{1 hour}

\section{1 week}
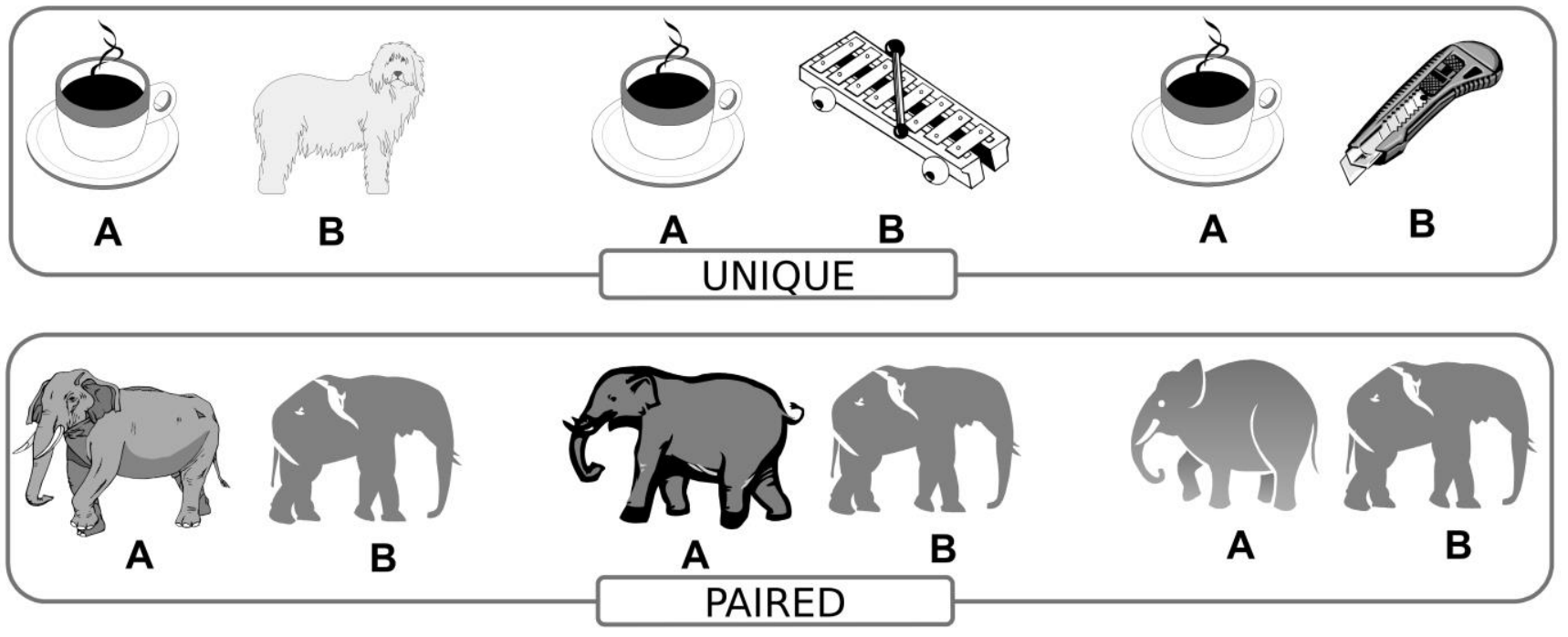

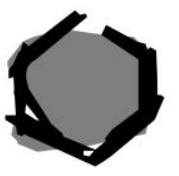

A

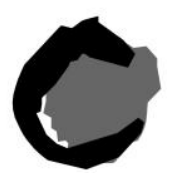

B

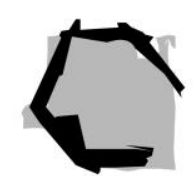

A

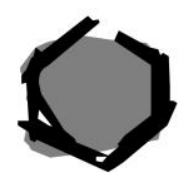

B

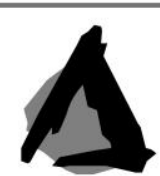

A

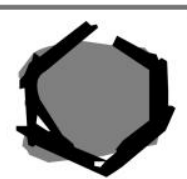

B 